\title{
Unusual conductance quantization and possible nuclear spin order- ing in a semiconductor
}

Evidence for Helical Nuclear Spin Order in GaAs Quantum Wires

Authors: C. P. Scheller, T.-M. Liu, G. Barak, A. Yacoby, L. N. Pfeiffer, K. W. West, and D. M. Zumbühl

arXiv: 1306.1940

\section{Recommendation and Commentary by Leon Balents, KITP, UCSB}

Nuclei play a pervasive but not the leading role in most solid state physics. They often have internal degrees of freedom - spin or quadrupole moments - which can be probed by external fields, and also serve as interrogators for electronic physics. They interact, however, relatively weakly with the rest of a solid, as well as with external fields (because they carry very small magnetic moments), which tends to make them a probe rather than a participant in the physics of solids. That aspect is the basis of most NMR physics and technology.

In semiconductors, nuclear interactions with electrons have been the focus of considerable recent work. They were recognized as one of the main sources of decoherence for electron spins confined in quantum dots. They provide, via hyperfine interactions, local fields which act on such electrons, and which are not easily removed. An interesting feature of this problem is that there are a very large number of nuclear spins per electron, which to a good approximation do not interact with one another except through the electron. This defines a "central spin" problem of considerable intrinsic interest. By utilizing this interaction, it has also proven possible to manipulate nuclear spins into interesting non-equilibrium states, for example "pumping" them into polarized configurations by selectively interacting with the electrons, which themselves feed polarization to nuclei.

In the paper above, the authors make a case for an equilibrium ordering of nuclear spins induced by such electron-nuclear coupling. The experimenters study double quantum wires created by cleaved edge overgrowth in a GaAs/AlGaAs heterostructure (see Fig.1 of the paper). Manipulating a gate electrode, they progressive deplete a pair of parallel $2 \mu \mathrm{m}$ wires, observing the characteristic conductance steps when the number of modes in those wires is small. The focus is on the upper wire, which has adiabatic contacts to a two 
dimensional electron gas that serves as leads, and hence is expected to display conductance quantization in units of $2 e^{2} / h$. The factor of 2 here arises from electron spin degeneracy. Below $100 \mathrm{mK}$, they observe a pronounced and repeatable plateau of conductance $e^{2} / h$. This suggests a lifting of the spin degeneracy, and the authors compellingly argue against a plethora of other explanations. Most of the latter were developed to explain the much-studied "0.7 structure" seen in quantum point contacts, which shows some similarity to the effect seen here. However, the new observation is much more robust, and has the opposite temperature dependence to the 0.7 structure.

The idea of magnetic ordering in one dimension has its challenges. For example, for a repulsively interacting, single subband electron gas, Lieb and Mattis proved long ago that the ground state must be a spin zero state. But the electron-nuclear coupling makes the Hamiltonian into a particular limit of a Kondo lattice, which is not covered by the Lieb-Mattis theorem. After a careful consideration by the authors of other possible explanations, the last man standing, theoretically, seems to be remarkably simple. According to a proposal by Braunecker, Simon, and Loss[1], RKKY interactions mediated by the hyperfine interaction drive the formation of a spiral ordering of the nuclear spins at the $2 k_{F}$ wavevector of the electrons. In turn, the exchange field from the ordered nuclei opens a gap for one branch of electronic excitations. This proposal appealing explains the insensitivity to fields, and roughly the right temperature scale for the experiment. While conceptually simple, the idea is audacious and I for one am amazed it might be true!

The proposed ordering is an equilibrium thermodynamic phenomena, very distinct from the non-equilibrium nuclear polarization studied quite intensively in early studies in semiconductor structures. It would not quite be the first example of nuclear magnetic ordering observed experimentally, but prior examples are few and occur at very low temperature: nanokelvin nuclear antiferromagnetism in $\mathrm{Ag}$ and $\mathrm{Cu}, 35 \mu \mathrm{K}$ ferromagnetic ordering of In nuclei in $\mathrm{AuIn}_{2}$. Probably the closest example to the current one is $2.5 \mathrm{mK}$ combined nuclear and electronic ferromagnetism in $\mathrm{PrCu}_{6}$. If the interpretation of the current experiment proves correct, spontaneous nuclear magnetism is occurring at a temperature 50 times higher here. One should note that there is some subtlety due to fluctuation effects common to one dimensional systems, but their effects are mitigated by the finite length of the wires and by a kind of averaging arising from the large number of nuclear spins coupling to each electron.

The connection of the present experiment to nuclear ordering is circum- 
stantial. A moderate applied magnetic field of a few Tesla had little or no effect on the observed $e^{2} / h$ plateau, which is consistent with a state where the nuclear exchange field is larger than the nuclear Zeeman energy. Also in particular a helix might be expected to smoothly deform into a polarized state anyway, without change of conductance (as opposed to a ferromagnet which presumably would orient to the field at small fields). Still, NMR measurements were attempted but nothing attributable to the proposed spin helix was observed. Thus there is room for skepticism on the interpretation, and likewise a need to seek more direct tests of nuclear involvement experimentally. Regardless, the observation is a dramatic one, and it is an impressive experiment.

\section{References}

[1] Bernd Braunecker, Pascal Simon, and Daniel Loss. Nuclear magnetism and electron order in interacting one-dimensional conductors. Phys. Rev. B, 80:165119, Oct 2009 . 